\title{
Indicações e Achados das Colonoscopias nos Doentes HIV-Positivo. Comparação com Soronegativos
}

\section{Indications and Results of Colonoscopies In HIV-Positive Patients. Comparison with Seronegative Patients}

\author{
CARMEN RUTH MANZIONE ${ }^{1}$; SIDNEY ROBERTO NADAL $^{2}$; \\ THIAGO DA SILVEIRA MANZIONE ${ }^{3}$; FERNANDA RIBEIRO ITO ${ }^{3}$ \\ ${ }^{1}$ Responsável pelo Serviço de Colonoscopia do Instituto de Infectologia Emilio Ribas; ${ }^{2}$ Chefe da Equipe Técnica de \\ Proctologia do Instituto de Infectologia Emilio Ribas; ${ }^{3}$ Ex-Estagiário do Serviço de Colonoscopia do Instituto de \\ Infectologia Emilio Ribas - São Paulo - SP - Brasil.
}

MANZIONE CR; NADAL SR; MANZIONE TS; ITO FR. Indicações e Achados das Colonoscopias nos Doentes HIV-Positivo. Comparação com Soronegativos. Rev bras Coloproct, 2007;27(1): 026-030.

RESUMO: Embora as indicações da colonoscopia sejam semelhantes em doentes HIV-positivo, quando comparados aos soronegativos, os achados e as suas incidências parecem diferir, sendo alguns deles mais específicos para o grupo dos imunodeprimidos. Objetivo: Avaliar as indicações e os achados das colonoscopias comparando os doentes soropositivos com os soronegativos para o HIV. Realizamos 1909 exames entre 1997 e 2005, sendo 1341 HIV-negativo (771 mulheres e 570 homens com média etária de 51,2 anos) e 568 HIVpositivo (137 mulheres e 431 homens com 34,4 anos, em média). A análise estatística dos dados estudados revelou haver mais exames em homens no grupo HIV-positivo e em mulheres entre os soronegativos. O grupo mais jovem foi o dos soropositivos. Houve mais indicações devidas a enterorragia $(\mathbf{2 2 , 1 \%})$ e dor abdominal $(\mathbf{1 2 , 7 \%})$ nos pacientes soronegativos, e diarréia crônica (45,9\%) entre os soropositivos. As colites e os tumores foram os achados mais comuns em pacientes HIV-positivo e a moléstia diverticular e os pólipos, os mais freqüentes entre os soronegativos. $O$ tumor predominante nos soronegativos foi o adenocarcinoma, enquanto que entre os soropositivos foi o sarcoma de Kaposi. Conclusão: A comparação entre os grupos demonstrou haver diferenças com relação ao sexo, idade e algumas indicações. Os achados foram semelhantes, embora com freqüências distintas.

Descritores: Colonoscopia; Diarréia; Pólipos; Sangramento gastrintestinal; Infecções por HIV; AIDS.

\section{INTRODUÇÃO}

A colonoscopia tem importância no arsenal diagnóstico dos portadores de sintomas colorretais, permitindo diagnosticar doenças e seus agentes etiológicos. Está indicada para pesquisa de sangramento digestivo, para diagnóstico diferencial das diarréias e das estenoses colônicas, para avaliar tumores abdominais e para detecção e seguimento de pólipos, câncer e colites. ${ }^{1}$ Também pode ser utilizada em portadores do vírus da imunodeficiência humana (HIV), uma vez que várias afecções e síndromes colorretais, oportunistas ou não, acometem esses doentes. ${ }^{2-6}$ Alguns autores relataram que, dentre aqueles com queixas intestinais, a diarréia crônica ocorria entre 50 e $80 \%$, a dor abdo- minal em 10 a 30\%, a hemorragia digestiva em $10 \%$ e outros sintomas como a constipação intestinal alternada ou não com diarréia em $10 \%$. $^{3}$

As contra-indicações para sua realização em doentes com AIDS, também são semelhantes às dos doentes soronegativos. ${ }^{2}$ Elas incluem peritonite, doença inflamatória aguda e distúrbios de coagulação, e devem ser evitadas em doentes não colaborativos ou em mau estado geral. ${ }^{2,3}$ Embora seja segura, a colonoscopia não é isenta de complicações mesmo em mãos experientes. Apesar da pequena incidência, são mais comuns no exame com finalidade terapêutica que para diagnóstico. As mais encontradas são o sangramento e a perfuração, geralmente identificados durante o próprio exame, e os problemas

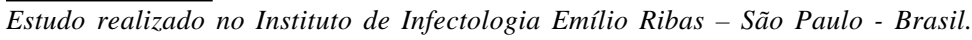

Recebido em 11/11/2006

Aceito para publicação em 08/02/2007 
cardiopulmonares. ${ }^{1,7}$ A bacteremia, que pode levar ao choque séptico, é a complicação mais grave. A avaliação clínica e laboratorial selecionará aqueles em que o procedimento poderá ser feito com segurança. ${ }^{7-9}$

Embora as indicações sejam semelhantes em doentes HIV-positivo, quando comparados aos soronegativos, os achados e a incidência dos mesmos parecem diferir, sendo alguns deles específicos para o grupo dos imunodeprimidos.

O objetivo do nosso estudo foi avaliar as indicações e os achados das colonoscopias comparando os doentes HIV-positivo com os HIV-negativo.

\section{MÉTODO}

Realizamos 1909 exames entre 1997 e 2005, excluindo aqueles com preparo inadequado e com contra-indicações clínicas. Eram 1341 HIV-negativo (771 mulheres e 570 homens com média etária de 51,2 anos) e 568 HIV-positivo (137 mulheres e 431 homens com 34,4 anos, em média). Os preparos de cólon foram feitos com lactulose e bisacodil. Antes do início do exame, realizamos o toque retal e caso houvesse fezes na ampola retal, consideramos o preparo como inadequado e suspendemos o exame. As indicações mais comuns nos doentes HIV-positivo foram diarréia crônica, estadiamento para sarcoma de Kaposi, enterorragia e pesquisa para citomegalovírus (CMV).(Tabela 1) Já, entre os HIV-negativo foram enterorragia, dor abdominal, constipação intestinal, alteração do hábito intestinal, diarréia crônica e controle pós-operatório de câncer colorretal (Tabela 2.)

\section{RESULTADOS}

Durante a colonoscopia, o preparo foi considerado insuficiente para o exame adequado e o diagnóstico preciso em $112(8,4 \%)$ doentes HIV-negativo e em 87 (15,3\%) HIV-positivo. O teste do qui-quadrado revelou maior incidência de mau preparo identificado durante o exame nos doentes soropositivos para o HIV. ( $p=0,00001)$ Nas indicações por diarréia crônica, os achados mais comuns em doentes HIV-positivo foram exames normais $(39,5 \%)$ e colites $(19,5 \%)$ e nos HIV-negativo foram exames normais $(54,4 \%)$, moléstia diverticular $(11,4 \%)$ e colites $(9,6 \%)$. Nas enterorragias, os achados foram exames normais $(40,2 \%)$ e tumores $(9,8 \%)$ e colites $(7,3 \%)$ para os HIV- positivo e exames normais (37,8\%), moléstia diverticular $(20,3 \%)$ e pólipos $(10,8 \%)$ nos soronegativos. Nos casos de dor abdominal, os exames foram normais em $37,5 \%$ e $52,6 \%$; colites em $12,5 \%$ e $0,6 \%$; tumores em $10 \%$ e $2,3 \%$ e moléstia diverticular em $2,5 \%$ e $22,2 \%$, respectivamente em soropositivos e negativos. (Tabelas 3 e 4$)$

A análise estatística dos dados estudados revelou haver mais exames em homens no grupo HIVpositivo, e em mulheres entre os soronegativos. A faixa etária foi mais jovem entre os soropositivos. Houve mais indicações devidas a enterorragia e a dor abdominal nos doentes soronegativos, e por diarréia crônica entre os soropositivos. As colites e os tumores foram os achados mais comuns em doentes HIV-positivo, e a moléstia diverticular e os pólipos foram mais freqüentes entre os soronegativos. O tumor predominante nos soronegativos foi o adenocarcinoma, enquanto que entre os soropositivos foi o sarcoma de Kaposi.

Tabela 1 - Distribuição das indicações de colonoscopia de 1341 doentes HIV-negativo. Instituto de Infectologia Emilio Ribas, 2006.

\begin{tabular}{lcc}
\hline Indicações & $\mathbf{n}^{\mathbf{0}}$ & $\mathbf{( \% )}$ \\
\hline Enterorragia & 321 & $(23,9)$ \\
Dor abdominal & 201 & $(15,0)$ \\
Obstipação & 141 & $(10,5)$ \\
Alteração do hábito intestinal & 137 & $(10,2)$ \\
Diarréia crônica & 124 & $(9,2)$ \\
Controle de câncer colorretal & 114 & $(8,5)$ \\
Outros & 303 & $(22,6)$ \\
Total & 1341 & $(100)$ \\
\hline
\end{tabular}

Tabela 2 - Distribuição das indicações de colonoscopia de 568 doentes HIV-positivo. Instituto de Infectologia Emilio Ribas, 2006.

\begin{tabular}{lrc}
\hline Indicações & $\mathbf{n}^{\mathbf{0}}$ & $\mathbf{( \% )}$ \\
\hline Diarréia crônica & 315 & $(55,4)$ \\
Estádio de sarc. de Kaposi & 98 & $(17,2)$ \\
Enterorragia & 93 & $(16,4)$ \\
Pesquisa para CMV & 31 & $(5,5)$ \\
Outros & 31 & $(5,5)$ \\
Total & 568 & $(100)$ \\
\hline
\end{tabular}


Tabela 3 - Distribuição das indicações e dos achados das 1341 colonoscopias realizadas em doentes HIVnegativo. Instituto de Infectologia Emilio Ribas. 2005.

\begin{tabular}{|c|c|c|c|c|c|c|}
\hline & Enterorr. & dor abd & diar crôn & obstip & AHI & po neo \\
\hline Colono nl & $(37,8)$ & $90(52,6)$ & $62(54,4)$ & $57 \quad(44,2)$ & $61(48,8)$ & $62(58,5)$ \\
\hline Prep inadequado & $38 \quad(12,8)$ & $15(08,8)$ & $13(11,4)$ & $24 \quad(18,6)$ & $14(11,2)$ & $8(07,6)$ \\
\hline Mol. Diverticular & $60 \quad(20,3)$ & $38(22,2)$ & $13(11,4)$ & $11 \quad(08,5)$ & $31 \quad(24,8)$ & $10(09,4)$ \\
\hline Tumores & $26 \quad(08,8)$ & $4(02,3)$ & $2(01,7)$ & $4 \quad(03,1)$ & $6(04,8)$ & $5(04,7)$ \\
\hline Pólipos & $32 \quad(10,8)$ & $11(06,4)$ & $2(01,7)$ & $6 \quad(04,7)$ & $7(05,6)$ & $7(06,6)$ \\
\hline Colites & $11 \quad(7,3)$ & $1 \quad(0,6)$ & $11(09,7)$ & $(02,3)$ & $1 \quad(0,8)$ & $0 \quad(0,0)$ \\
\hline Total & 296 & 171 & 114 & 129 & 125 & 106 \\
\hline
\end{tabular}

Tabela 4 - Distribuição das indicações e dos achados das 568 colonoscopias realizadas em doentes HIVpositivo. Instituto de Infectologia Emilio Ribas. 2005.

\begin{tabular}{|c|c|c|c|c|c|c|}
\hline & Enterorr. & dor abd & diar crôn & pesq de tu & estad SK & pesq CMV \\
\hline Colono nl & $33 \quad(40,2)$ & $15(37,5)$ & $103(39,5)$ & $6(31,6)$ & $40(48,1)$ & $11(42,2)$ \\
\hline Prep inadequado & $12(14,6)$ & $10(25,0)$ & $44(16,9)$ & $3(15,8)$ & $12(14,1)$ & $6(23,1)$ \\
\hline Mol. Diverticular & $0 \quad(00,0)$ & $1(02,5)$ & $0(00,0)$ & $1 \quad(05,3)$ & $0 \quad(0,0)$ & $0 \quad(0,0)$ \\
\hline Tumores & $8 \quad(09,8)$ & $4(10,0)$ & $0 \quad(0,0)$ & $5 \quad(26,3)$ & $13(15,3)$ & $2(07,7)$ \\
\hline Pólipos & $0 \quad(0,0)$ & $2(05,0)$ & $0 \quad(0,0)$ & $4 \quad(21,0)$ & $1(01,2)$ & $0 \quad(0,0)$ \\
\hline Colites & $6(07,3)$ & $5(12,5)$ & $51(19,6)$ & $1 \quad(05,3)$ & $2(02,4)$ & $5(19,2)$ \\
\hline Total & 82 & 40 & 261 & 19 & 85 & 26 \\
\hline
\end{tabular}

\section{DISCUSSÃO}

Alguns exames são utilizados para avaliar o doente HIV-positivo antes de realizar o procedimento endoscópico. A contagem de linfócitos T CD4+ deve ser superior a $200 / \mathrm{mm}^{3}$, ou quando abaixo desse número, os leucócitos devem ser superiores a 3.000/ $\mathrm{mm}^{3} ;{ }^{10}$ as plaquetas devem estar acima de $50.000 / \mathrm{mm}^{3}$; a hemoglobina superior a $10 \mathrm{~g} / \mathrm{dl}$ e o tempo de protrombina maior que $60 \% .{ }^{11}$ Todavia, em alguns casos selecionados, podemos realizar o exame mesmo existindo exames com resultados adversos. Por exemplo, em doentes com hemoglobina baixa e sem dispnéia a colonoscopia poderá ser realizada, já em doentes dispnéicos, algumas vezes se contra-indica o exame mesmo com valores maiores de hemoglobina, devido à possibilidade de piorar o quadro pulmonar. Maior incidência de diagnósticos pode ser esperada em doentes com febre, perda de peso e contagens de linfócitos $\mathrm{T}$ CD4 inferiores a $200 / \mathrm{mm}^{3}$, especialmente se abaixo de $50 / \mathrm{mm}^{3}{ }^{12}$
Para o exame é necessário preparo intestinal. Os vários métodos conhecidos podem ser aplicados, conforme a preferência do profissional. Utilizamos método anterógrado com lactulose e bisacodil, com bons resultados. ${ }^{13} \mathrm{Na}$ véspera do exame, o doente é orientado ingerir dieta sem resíduos ou corantes no almoço, e caldo de carne coado no jantar, além de líquidos à vontade. O bisacodil, quatro comprimidos, é tomado com 12 horas de antecedência. A ingestão de $120 \mathrm{ml}$ de lactulose diluídos para um litro de líquido (água, chá, suco de laranja coado) deve ocorrer em uma hora, seis horas antes da colonoscopia. O doente é aconselhado a beber mais um litro de líquidos até a hora do exame para se manter hidratado. Preferimos a lactulose a outros laxantes osmóticos porque o produto é de fácil aquisição, sendo vendido em farmácias, e o preparo pode ser domiciliar. O tempo longo de preparo, de 4 a 6 horas, proporciona menor risco de distúrbio hidroeletrolítico. O produto é eficaz, com bons resultados em $87 \%$ dos doentes e com intolerância ao 
produto em apenas $3,5 \%$, caracterizada por náuseas e vômitos. ${ }^{13}$ Além disso, pode ser utilizável em estenoses parciais, sem dano ao doente. ${ }^{13}$

A hemorragia é a complicação mais comum e ocorre imediatamente após biópsias e polipectomias. As perfurações surgem durante os mesmos procedimentos e são diagnosticadas mais precocemente quando a lesão está no ceco. Pode haver perfuração durante colonoscopia diagnóstica e sendo mais comuns no cólon esquerdo. ${ }^{7}$ A bacteremia é definida pelo encontro de germes da flora intestinal na corrente sanguínea. ${ }^{8}$ Esse achado pode ser verificado cinco minutos após o início do procedimento endoscópico. ${ }^{9}$ Entretanto, o início dos sintomas ocorre desde durante o exame até 12 horas após. ${ }^{14}$ Acredita-se que a compressão intrínseca pelo ar injetado pode causar a translocação bacteriana, ou seja, a passagem de bactérias da luz intestinal para a corrente sanguínea e linfática. ${ }^{15}$ Isso pode ocorrer na presença de solução de continuidade da mucosa colônica, incluindo biópsias, ${ }^{14}$ embora outros observassem que esses fatores não constituíram risco para bacteremia. ${ }^{8}$ Provavelmente esteja mais ligada à deficiência do sistema imune gastrointestinal que à desinfecção do endoscópio. ${ }^{16}$ Entretanto, o estudo do efeito da distensão colônica na translocação bacteriana sugeriu que ocorre ativação do sistema de coagulação por dilatação dos vasos intestinais intramurais e não pelas endotoxinas que escapam do lúmen. Os fatores de risco incluem uso de drogas intravenosas, presença de cateter venoso central, neutropenia ${ }^{17}$ e contagem de linfócitos T CD4+ inferior a $100 / \mathrm{mm}^{3}{ }^{18,19} \mathrm{O}$ esquema antiviral com inibidores da protease determinou redução da incidência e modificação das ca- racterísticas da bacteremia. ${ }^{17} \mathrm{Na}$ população em geral, a incidência está em torno de $0 \%$ a 5\%, independente de biópsia ou polipectomia. ${ }^{8,9,16,20}$ Os enterococos são os mais encontrados e a profilaxia deve ser realizada contra esses microorganismos. ${ }^{21}$ Todavia, a presença de sintomas é rara ${ }^{22}$ e também é duvidosa a eficácia da profilaxia antimicrobiana para todos os casos. ${ }^{8,14,22}$ Bacteremia e endocardite por Streptococcus bovis parecem estar associadas à presença de neoplasmas malignos do cólon e de doença hepática. $^{23}$

A Associação Americana dos Cirurgiões do Cólon e Reto indica o uso profilático de antimicrobianos em algumas situações consideradas de risco, entre elas em portadores de valvulopatia, prótese cardíaca e outras próteses vasculares ou ortopédicas. ${ }^{24}$ Além disso, alguns estudos sugerem que doentes com quadro séptico de origem colorretal ou de outros locais, bem como os portadores de hepatopatia crônica com ascite, têm maior risco para desenvolver bacteremia póscolonoscopia. ${ }^{25,26}$ Acreditamos que esses critérios devam ser estendidos aos doentes imunodeprimidos, incluindo os doentes HIV-positivo. $\mathrm{O}$ fato de alguns deles terem imunodeficiência acentuada nas fases mais avançadas da doença poderia ser motivo de maior incidência de bacteremia.

A colonoscopia, obedecendo aos parâmetros descritos, é exame útil e seguro para diagnóstico das doenças que acometem os portadores da infecção pelo HIV.

A comparação entre os grupos demonstrou que houve diferenças com relação ao sexo, idade e algumas indicações. Os achados foram semelhantes, embora com freqüências distintas.

\footnotetext{
ABSTRACT: Although colonoscopy indications are similar in HIV-positive patients when compared to sero-negative persons, the findings and their incidences seem to be different, and some are more specific to immunosuppressed patients. The aim of this study is to evaluate indications and findings of colonoscopies comparing patients, sero-negatives and sero-positives to HIV. We performed 1909 colonoscopies from 1997 to 2005, 1341 in HIV-negative persons (771 females and 570 males with mean age of 51.2 years old) and 568 in HIV-positive people (137 females and 431 males with mean age of 34.4 years old). Statistics revealed more males among HIV-positive patients and more females in the sero-negative group. The HIV-positive was the youngest group. There were more indications due to intestinal bleeding $\mathbf{( 2 2 . 1 \% )}$ and abdominal pain (12.7\%) in the HIVnegative persons, and chronic diarrhea $(\mathbf{4 5 . 9 \%})$ among the sero-positive patients. Colitis and tumors were the commonest findings in HIV-positive patients and diverticulosis were the most frequent among the sero-negative patients. The most diagnosed tumors were adenocarcinomas in the sero-negative group and Kaposi sarcoma among the sero-negative patients. The comparison between these two groups showed differences in gender, age and some indications. Findings were similar, despite incidences being different.
}

Key words: Colonoscopy; Diarrhea, Polyps; Gastrintestinal bleeding; HIV infections; AIDS. 


\section{REFERÊNCIAS}

1. Cappell MS, Friedel D. The role of sigmoidoscopy and colonoscopy in the diagnosis and management of lower gastrointestinal disorders: endoscopic findings, therapy, and complications. Med Clin North Am 2002;86:1253-88.

2. Manzione CR, Nadal SR. Colonoscopia no doente HIV-positivo. Rev bras Coloproct 2005;25(3):265-268.

3. Manzione CR, Nadal SR, Calore EE, Manzione TS. Achados colonoscópicos e histológicos em doentes HIV-positivo com diarréia crônica. Rev bras Coloproct. 2003;23:256-261.

4. Brink AK, Mahe C, Watera C, Lugada E, Gilks C, Whitworth $\mathrm{J}$ et al. Diarrhea, CD4 counts and enteric infections in a community-based cohort of HIV-infected adults in Uganda. J Infect 2002;45:99-106.

5. Navin TR, Weber R, Vugia DJ, Rimland D, Roberts JM, Addiss DG et al. Declining CD4+ T-lymphocyte counts are associated with increased risk of enteric parasitosis and chronic diarrhea: results of a 3-year longitudinal study. J Acquir Immune Defic Syndr Hum Retrovirol 1999;20:154-9.

6. Prasad KN, Nag VL, Dhole TN, Ayagari A. Identification of enteric pathogens in HIV-positive patients with diarrhea in northern India. J Health Popul Nutr 2000; 18: 23-6.

7. Dafnis G, Ekbom A, Pahlman L, Blomqvist P. Complications of diagnostic and therapeutic colonoscopy within a defined population in Sweden. Gastrointest Endosc 2001;54:302-9.

8. El-Baba M, Tolia V, Lin CH, Dajani A Absence of bacteremia after gastrointestinal procedures in children. Gastrointest Endosc 1996;44:378-81.

9. Botoman VA, Surawicz CM. Bacteremia with gastrointestinal endoscopic procedures. Gastrointest Endosc 1986;32:342-346.

10. Nadal SR, Manzione CR, Galvão VM, Salim VRBM, Speranzini MB. Perianal diseases in HIV-positive patients compared with a seronegative population. Dis Colon Rectum 1999;42:649-54.

11. Bonacini M, Skodras G, Quiason S, Kragel P. Prevalence of enteric pathogens in HIV-related diarrhea in the midwest. AIDS Patient Care STDS 1999;13:179-84.

12. Oldfield EC 3rd. Evaluation of chronic diarrhea in patients with human immunodeficiency virus infection. Rev Gastroenterol Disord. 2002;2:176-88.

13. Manzione CR, Nadal SR. Preparo domiciliar de cólon com bisacodil e solução de lactulose a $10 \%$ para colonoscopia ambulatorial. Rev bras Coloproct 2000;20:88-92.

14. Meyer GW, Artis AL. Antibiotic prophylaxis for orthopedic prostheses and GI procedures: report of a survey. Am J Gastroenterol 1997;92:989-991.

15. Schoeffel U, Jaeger D, Butt J, Salm R, Farhmann EH. Effect of human bowel wall distension on translocation of indigenous bacteria and endotoxins. Dig Dis Sci 1994;39:490-493.
16. Schembre DB. Infectious complications associated with gastrointestinal endoscopy. Gastrointest Endosc Clin N Am 2000;10:215-32.

17. Tumbarello M, Tacconelli E, Donati KG, Citton R, Leone F, Spanu T et al. HIV-associated bacteremia: how it has changed in the highly active antiretroviral therapy (HAART) era. J Acquir Immune Defic Syndr 2000;23:145-51.

18. Meynard JL, Guiguet M, Fonquernie L, Lefebvre B, Lalande $\mathrm{V}$, Honore I et al. Impact of highly active antiretroviral therapy on the occurrence of bacteremia in HIV-infected patients and their epidemiologic characteristics. HIV Med 2003;4:127-32.

19. Meyer CN, Skinhoj P, Prag J. Bacteremia in HIV-positive and AIDS patients: incidence, species distribution, risk-factors, outcome, and influence of long-term prophylactic antibiotic treatment. Scand J Infect Dis 1994; 26:635-42.

20. Low DE, Shoenut JP, Kennedy JK, Sharma GP, Harding GK, Den Boer B et al. Prospective assessment of risk of bacteremia with colonoscopy and polipectomy. Dig Dis Sci 1987;32:12391243.

21. Dajani AS, Bisno AL, Chung KJ, Durack DT, Freed M, Gerber MA et al. Prevention of bacterial endocarditis: recommendations by the American Heart Association. JAMA 1990;130:84-87.

22. Church JM. Infection and desinfection. In, Church MJ, Endoscopy of the colon, rectum, and anus. Igaku-Shoin. New York, Tokio. 1995, pp. 44-52.

23. Waisberg J, Matheus CO, Pimenta J. Infectious endocarditis from Streptococcus bovis associated with colonic carcinoma: case report and literature review. Arq Gastroenterol 2002;39:177-80.

24. American Society of Colon and Rectum Surgeons. Practice parameters for antibiotic prophylaxis to prevent infective endocarditis or infected prosthesis during colon and rectum endoscopy. Dis Colon Rectum 1992;35:277-85.

25. Thornton JR, Losowky MS. Septicaemia after colonoscopy in patients with cirrhosis. Gut 1991;32:450-451.

26. Zuckerman GR, O'Brien J, Halsted R. Antibiotic prophylaxis in patients with infectious risk factors undergoing gastrointestinal endoscopic procedures. Gastrointest Endosc 1994;40:538-543.

\section{Endereço para correspondência: CARMEN RUTH MANZIONE}

Rua Dr. Virgilio de Carvalho Pinto, 381 apto 23

São Paulo - Capital - CEP 05415-030

e-mail:srnadal@terra.com.br 